\title{
Design of Ship Anti-collision System Based On Microcontroller
}

\author{
Xiao-ling Liu ${ }^{1, a,{ }^{*}}$, Fu-zhen Qin ${ }^{1, b}$ and Hai-chun Niu ${ }^{1, c}$ \\ ${ }^{1}$ Qingdao Huanghai College, Qingdao, Shandong, China \\ a240120594@qq.com, b 765589715@qq.com, ' $582530799 @ q q . c o m$
}

\begin{abstract}
In the current transportation, the ship is a very important means of transport. Although the water transport traffic has unparalleled advantages of other traffic, the ship accident is also happening. Therefore, ship collision avoidance has been the core of research in various fields. This article uses the AT89C52 microcontroller as the core chip, through laser range finder measurement, displaying the corresponding information on the monitor, to develop a ship collision avoidance system.
\end{abstract}

Keywords: MCU, Ship, Avoidance, Laser range finder

\section{Introduction}

In contemporary society, the development of traffic is getting faster and faster, and various ships of different modes of transport have played a very important role in the previous society. They are still making great contributions to the further development of mankind. Although the water transport traffic has unparalleled advantages of other traffic, but the ship accident is also occurring, and the risk is greater. In the event of a ship collision accident, the result will be economic losses and environmental pollution, more serious will cause casualties on board personnel. Therefore, in the process of ship navigation how to avoid collision, to achieve collision avoidance and intelligent early warning is very necessary.

The system is through the laser range finder to measure the distance between the ship and the target, when the target and the ship reaches the set distance from the safety value of the time, the microcontroller will control the display sound and light alarm, the ship's staff according to the LCD display Data to carry out the corresponding operation, thus achieving the collision avoidance of the ship.

\section{The development of Ship collision avoidance technology}

The developed countries have studied the ship collision avoidance system in the mid-20th century, and now all countries have developed the corresponding products. There are now automatic radar plotter (APRA), AIS (automatic identification system) and collision avoidance expert system both at home and abroad.

APRA as a modern large ship for collision avoidance of the main instruments, the main feature is to capture the target, to determine the risk, timely alarm, the decision to determine the target has a certain performance, ARPA another major advantage is when the ship around Target more, the use of ARPA is very convenient, you can timely and accurate information on all the targets are displayed on the radar display, reducing the time required to manually draw, to achieve a safe avoidance. But APRA also has some shortcomings, one wave, rain and snow, near the same frequency radar and machine noise will have an impact on the performance of 
the radar, the second ship in the swing will also affect the radar performance, reliability and accuracy.

Automatic Identification System (AIS) is developed after ARPA navigational System, AIS has the following functions: Identification of ships, To assist tracking targets, Simplify the information communication, To provide additional information and avoid collisions. But there are also some problems: the ship can only be on both sides of the AIS displayed on the screen when only two ships have both installed AIS equipment, and in the condition of equipment is open, while it can't display the ship is not installed AIS; the prices of AIS equipment is relatively high, many small and medium-sized shipping have not the economic strength; Due to the limitations of AIS, the AIS cannot be used alone, must be equipped with corresponding ARPA.

Collision avoidance expert system is the forefront of ship collision avoidance in the field of the problem, domestic and foreign experts have made some achievements. The application of the comprehensive disciplines of the collision avoidance system, which involves artificial neural network, fuzzy mathematics and genetic algorithms, which presents a major challenge to the study of the system. Of course, the study of the collision avoidance expert system should follow the "sea collision rules", and to make the collision avoid the expert system to meet the different properties of the ship. At present, there is no perfect collision avoidance expert system, the system is currently in the research stage.

Because the current collision avoidance device are relatively expensive, it is difficult to popularize for general middle and small ship,. So it need to develop ship collision avoidance system low price relatively, and can meet the demand of small and medium-sized.

\section{The overall design of system}

The main function of the system is through the single-chip control, constantly emitting laser pulse, to detect the target around the ship, and then collect the information into the microcontroller, processed by the microcontroller, and then the ship and the target The distance between the objects and the speed information of the target are displayed on the liquid crystal display. If the distance between the ship and the target ship is less than the safe distance set by the system, the alarm unit device will sound and light alarm, the crew according to the sound and light alarm tips to adjust the operation of the ship, select a reasonable route to successfully avoid obstacles, To achieve a safe navigation.

The system needs to develop an electric pedestal, and by the single-chip control motor to the base to rotate horizontally, so that it can measure the ship within 360 degrees within the target, rather than one of the target, to expand the measurement range of the system. So that the environment around the ship can be better monitored, so that the probability of collision of ships has been greatly reduced. The system diagram of the active collision avoidance is shown in the fig.1.: 


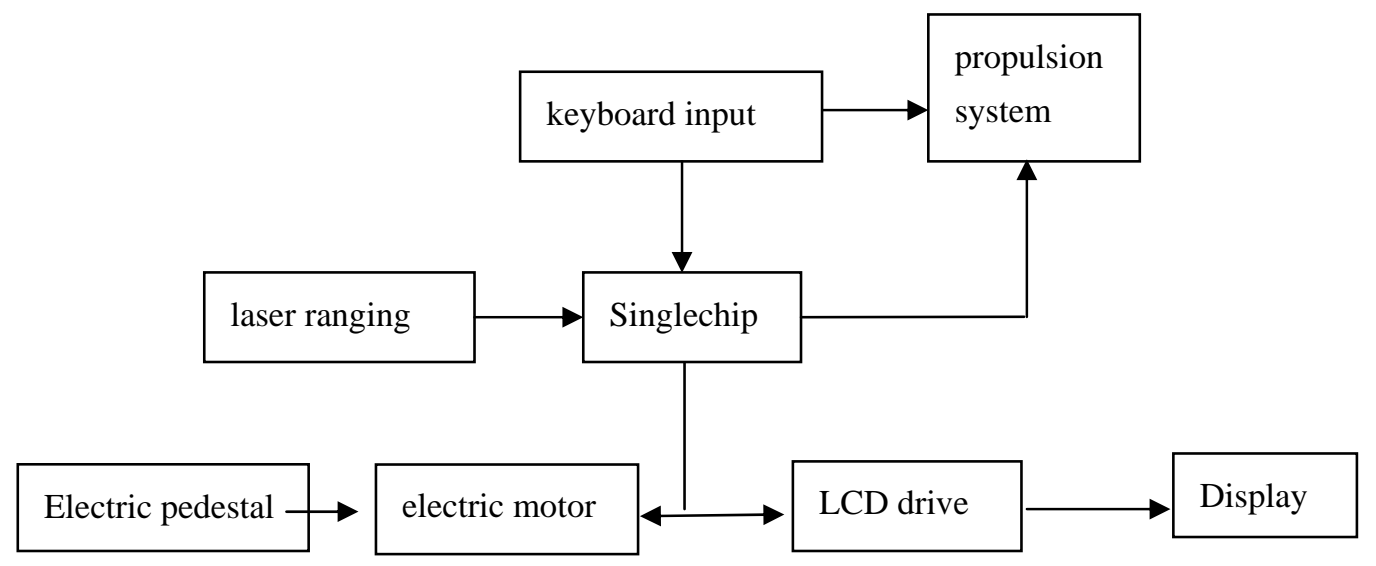

Fig 1 Block diagram of the ship active collision avoidance system

\section{The principle of laser ranging}

The laser range finder is an instrument that uses the laser to accurately measure the distance of the target. Laser range finder light weight, small size, easy to operate fast and accurate, the error is only one-fifth of the other optical range finder to a few percent.

Laser range finder generally uses two ways to measure the distance: pulse and phase method. At present, most of the laser ranging system using pulsed laser ranging method, the system uses laser pulse distance measurement method to achieve the distance between the ship and the ship.

Laser pulse ranging is the laser on the measured target to launch an optical pulse (the pulse width is generally less than $50 \mathrm{~ns}$ ), and then receive the system to receive the target reflection of the light pulse, by measuring the time of light pulse to calculate the target distance:

$$
S=\frac{c t}{2}
$$

In the Eq. 1, $\mathrm{S}$ representative measuring distance, $\mathrm{t}$ on behalf of the light pulse round-trip time, c on behalf of the velocity of propagation of light in a vacuum. In the laser pulse ranging, $\mathrm{t}$ is the first to determine the starting time, and then look at the starting time of the clock pulse swallow, to calculate the time t, schematic diagram is shown in figure 2.

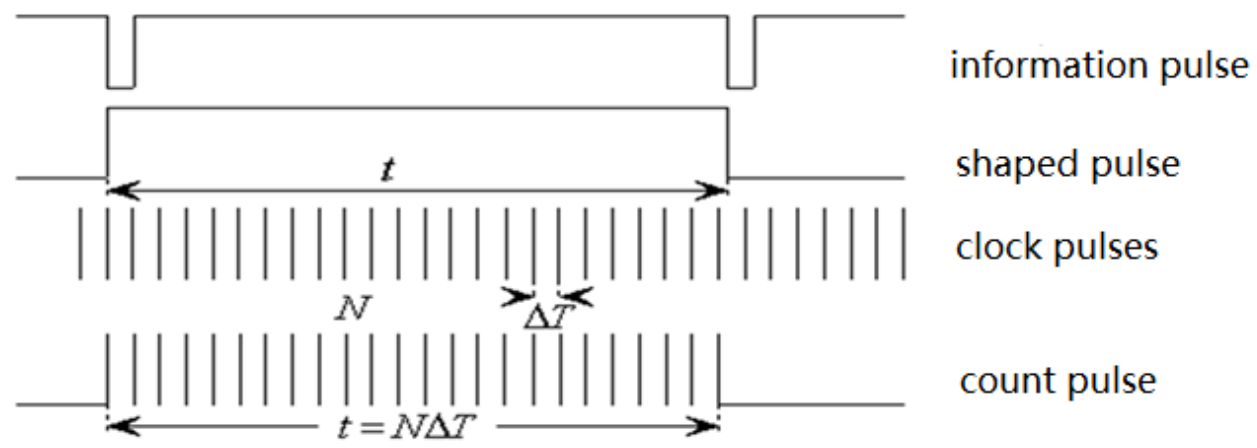

Fig 2 Laser pulse clocking principle

If you set the oscillation frequency of the clock pulse as $\mathrm{f}, \mathrm{N}$ clock pulse into the counter in t time, has established Eq. 2. 


$$
S=\frac{c}{2} N T=\frac{\mathrm{c}}{2 f} N=L N
$$

In Eq. 2, S represent the distance, and $\mathrm{L}$ represent each pulse distance, the size of $\mathrm{L}$ determines the accuracy of measurement of the pulse ranging system, the smaller the value of $\mathrm{L}$, the higher the precision. Such as number of clock pulse counter received is $\mathrm{N}, \mathrm{L}$ is varies according to the precision of range finder, can be calculated by Eq. 2 type $\mathrm{S}$ target distance.

\section{System hardware design}

The core of hardware is single chip microcomputer AT89c52 chip, the chip's main function is: $8 \mathrm{~KB}$ Flash program memory, $256 \mathrm{~b}$ data memory, three timers, 8 interrupt source.

Input part: The input part of the hardware consists of ordinary key switch, respectively, the implementation of the control system switch and mode of operation, as well as sound and light alarm circuit part of the selection mode and alarm release settings.

Display part: The display part of the hardware is composed of LED display, when the microcontroller to deal with the corresponding information, it can be displayed from the display, the information displayed generally includes the distance of the obstacle, height and the current system operating status.

Communication part: This part is mainly completed the data transmission, by the single-chip microcomputer issue instructions to the laser range finder, or by laser rangefinder data sent to the single-chip microcomputer. Communication part mainly composed of serial interface and MAX232 chip.

Dynamic execution part: This part is mainly composed of the motor drive part and the electric base part, by the single-chip to control the motor, so that the electric base can rotate horizontally, to complete the measurement of the surrounding objects.

Sound and light alarm part: The system is designed when the display shows the distance between the ship and the target is less than the system set the safety value, the system will sound and light alarm. This part is mainly composed of light-emitting diodes and buzzer, when the value is less than the safety value is displayed, the sound and light alarm circuit will send the corresponding light and sound to prompt the staff on board to select the appropriate operation.

\section{Conclusion}

This paper first studies the advantages and disadvantages of several collision avoidance devices commonly used in large ships, and then designs a collision avoidance system suitable for small and medium-sized ships based on laser range finder.

The system is based on the AT89C52 chip as the main circuit, the display shows the use of laser pulse distance measurement method to measure the ship near the target information, and the system also designed the display and sound and light alarm and other functions. In this paper, the hardware circuit is analyzed and designed, the next step need to build the actual experimental platform for verification.

\section{References}

[1] M.Inaish, et al: Basic research on a collision avoidance system using neutral networks. Proceedings IAIN91,1991. 
[2] Kai-Wen SU. A study on the expert system for ship collision avoidance. Dalian: Dalian Maritime University.2007.

[3] Jin-Song ZHAO, Feng-Chen WANG. The Ship collision avoidance learning principle. Dalian: Dalian Maritime University Press.1999.

[4] Fujii Y, Tanaka K. Traffic Capacity. Journal of Navigation, 1971,24(4):543-552. 